\title{
Dynamic models of team rowing for a virtual environment rowing training system
}

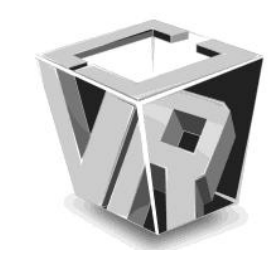

\author{
Alessandro Filippeschi ${ }^{1}$, Emanuele Ruffaldi ${ }^{1}$, Antonio Frisoli ${ }^{1}$, Carlo Alberto Avizzano ${ }^{1}$, Manuel Varlet ${ }^{2}$, \\ Ludovic Marin ${ }^{2}$, Julien Lagarde ${ }^{2}$, Benoit Bardy ${ }^{2}$, and Massimo Bergamasco ${ }^{1}$
}

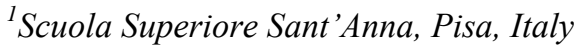

${ }^{2}$ University Montpellier 1, Montpellier, France

\begin{abstract}
In this paper we report on the strategies adopted to model team rowing behavior for an in-door rowing training system. This system allows athletes to train at in-door location while preserving the main features of out-door rowing. This paper focuses on the dynamic models employed to simulate out-door rowing, as well as on the modeling of team behavior, in order to set up visual feedback for team rowing training. These models are employed for controlling the output in the virtual environment, in particular for training the inter-rower synchronization in a team.
\end{abstract}

Index Terms-Biomechanics, dynamics, simulation, team rowing, training.

\section{INTRODUCTION}

The goal of each professional rower is to win races. Often the gap between the winning crew and the second one is less than a couple of seconds, while the entire race lasts typically between 5 and 8 minutes. Hence, it is clear how small improvements are important for winning a race, and that every factor determining the overall performance should be taken into account during rowing training. Many abilities underlie the rower's performance: the fitness status of the athletes [13], their technique, their intra- and inter-individual coordination, the ability to manage their energy stocks, as well as their ability to cope with a race $[9,12,13]$.

Although rowing training protocols are mainly focused on the rower's fitness status, rowing coaches and athletes have always looked for the best technique, aiming at the most efficient stroke. Technique is not the only issue for a winning crew: the coordination of the teammates is equally important, and it may become the main issue for a professional rowing team.

While the fitness status is continuously trained and checked, technique and coordination analyses are mainly carried out in out-door rowing, since the most employed in-door rowing simulators do not allow the user to reproduce all the features of the out-door rowing experience. Therefore, when the weather conditions do not allow out-door training, it is not possible to carry out a complete rowing training.

Moreover, technique and coordination analyses are mainly based on films of outdoor performance, while a real time

Manuscript received on 22 December 2008

E-Mail: a.filippeschi@sssup.it feedback is entrusted to the coach ability to detect the mistakes.

Such issues are the motivations for developing an in-door system capable of a complete rowing training in real time: a system that monitors, analyzes and gives feedback about the user's technique, allows the user to train the coordination with other teammates and allows him/her to cope with various opponents race strategies.

The challenge of rowing training has been undertaken within the SKILLS project [21], and it has been approached by means of the SKILLS methodology: expert performances are analyzed in order to understand what basic skills underlie the gesture. A multimodal system is designed to transfer these abilities to non-skilled users, while taking into account the capacity differences between novices and experts.

Many questions arise with respect to accomplishing such a task: what are the relevant variables to be captured, what are the best perceptual channels to give suitable feedback to the user, how should the appropriate training protocols for transferring the expert's skills to the common user be developed.

After a short review of the existing rowing simulators and the current training strategies, the system architecture of our simulator is briefly presented. We then focus on (i) the dynamic models employed for analyzing the user performance, (ii) how the team rowing behavior is modeled, and (iii) the guidelines for the development of a training protocol for rowing.

\section{STATE OF THE ART}

Many devices have been developed for rowing training. The most employed rowing simulator is the Concept $2^{\circledR}$ ergometer [4]. All rowing national federations around the world use it. The athlete's physical skills are evaluated using this device. While it is a good tool for the workout and the development of energetic resources, it is not appropriate for rowing technique and team training, since it does not allow the user to correctly reproduce the real gesture. Other devices, Biorower [2] and Oartec [16] for example, address this issue and are comfortable for the user, but they only output the user overall performance (speed, elapsed time, etc.), without deeply investigating the user's movement and timing. Devices that are more complex than the ones mentioned above are being developed by research institutes, but are not on the market yet. Two examples are the rowing simulator developed by the $\mathrm{M}^{3}$ lab of the Eidgenössische Technische Hochschule [5], and the simulator 
developed by the Ecole des Mines de Nantes [14]. The ETH simulator is composed of a real boat immersed in a $3 \mathrm{D}$ virtual environment projected onto four screens, where both visual and acoustic feedback is provided to the user. Two cables pull the oar shaft in order to replicate the resistance due to the blade-water interaction. The EMN simulator is composed of a light platform placed in front of a curved screen where a typical rowing scenario is projected.

Each rowing federation has its own training protocol, focused mostly on fitness training. Specific technique training sessions are seldom performed separately, and are often the last part of fitness training sessions. However, there are many databases of technique training exercises. Rowing manuals [7, 13, 23] show the typical technique errors of beginners and intermediate rowers, and propose specific exercises in order to correct them.

The same stands for team rowing: specific training sessions are not performed alone, but some typical errors have been detected and specific exercises have been set up to correct them.

Poor synchronization between rowers causes additional movements of the boat, including yawing, rolling and pitching [25]. It produces a decrease of power output and a waste of effort because of increased friction. All rowers have to learn how to synchronize their movements with the others. Even professional rowers have to learn this synchronization skill when constituting a new team. For teaching how to synchronize with a teammate, two possibilities are available to coaches: the "natural" situation (out-doors on the boat) or the simulator situation. The natural situation is mostly based on verbal feedbacks provided by the coach, which is rarely precise enough and producing an adequate level of synchronization takes time. However, it is often used because rowers directly adjust their movement in the natural environment. The second way uses the rowing simulator. The disadvantage of simulators is that water and boat movements are neglected or unrealistic, and it sometimes requires an extra period of adaptation to adjust inter-rower synchronization. However, more and more coaches have realized that simulators can accelerate the learning of synchronization. Simulators increase mechanical and visual interaction between rowers, and may enhance crew synchronization faster than in the natural context. In simulators, rowers are often sitting side-by-side instead of front-to-back as in a real boat. The interaction between rowers can be clearly felt and seen, and for this reason may be increased. Minor differences in coordination between crewmembers can thus be eliminated on land more effectively than on water. Generally speaking, however, the nature and frequency of synchronization feedback are rather poor, and no experimental study has ever validated their usefulness in the teaching of team rowing. This is one goal of our rowing training system (see below).

\section{SYSTEM ARCHITECTURE}

The rowing training system developed within the SKILLS project is shown in Fig. 1. It is composed of a mechanical platform, a set of sensors, a system for data reading, elaborating and storage, and a 40" LCD screen for visual feedback [18].

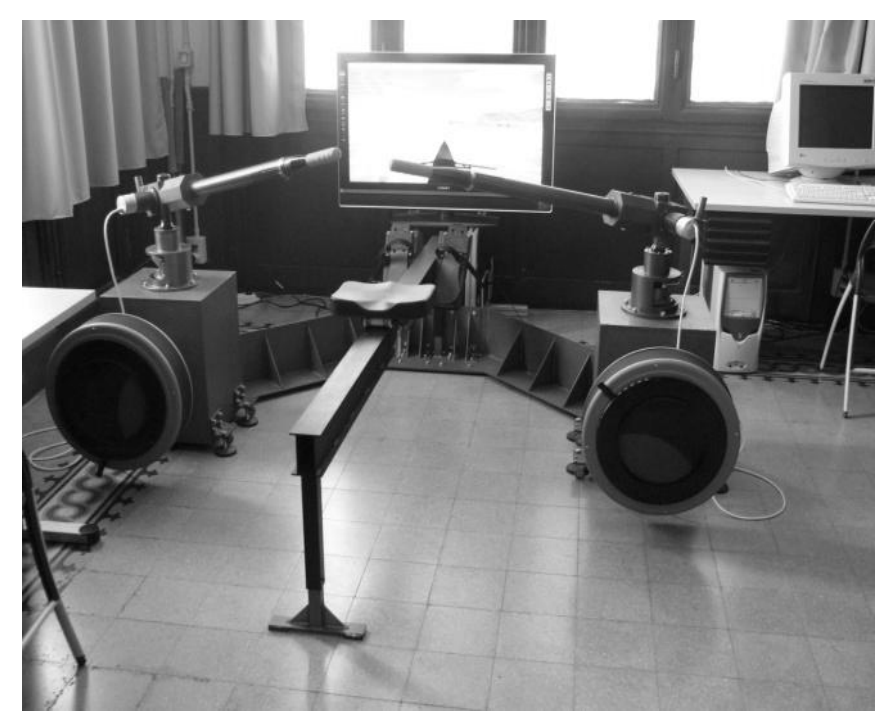

Fig. 1. The SKILLS rowing demonstrator

The sensors embedded in the system capture user's motion and exerted forces. Acquired data are read and elaborated by the software system, which also manages the storage and the feedback to the user. The system architecture is shown in Fig. 2.

The mechanical platform allows users to reproduce the out-door features of the rowing movements. Users of different sizes can comfortably perform both sculling and sweep rowing thanks to the regulation loops available. The resistance is provided by two Concept $2{ }^{\circledR}$ ergometer energy dissipaters, which allow replication of the same dependence of the resistant force on the oar kinematics.

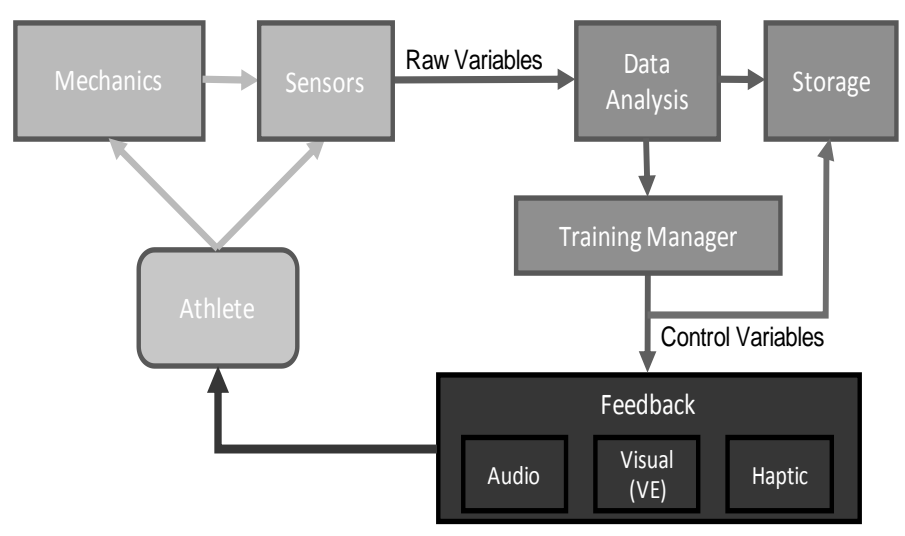

Fig. 2. The system architecture

Oar kinematics is read by two encoders that provide feedback about the position of the hands. The user's motion is then completed by the measurement of the seat and the head position respectively by means of an infrared position sensor and a webcam. Only the forces on the oars' handles are captured. They are obtained by directly measuring the torque on the shaft, which bears the oar, and by an analytical model of the energy dissipater [8]. 
Data acquired are the input for an analytical model of the racing boat, the hull, and the human models (see section 4). The boat model gives, as output, the boat motion and the technique efficiency. All data are then analyzed in order to obtain measures of performance. Motion data are employed to evaluate timing and coordination. Boat motion allows the estimation of energy expenditure. User and boat motion as well as performance analysis are further elaborated to give the user suitable feedback.

The LCD screen displays a virtual environment (VE) composed of a boat moving across a typical rowing scenario [8]. A rowing basin is surrounded by hills, the boat motion is determined by the boat's analytical model output. The virtual oars are synchronized with the real ones, while the point of view follows the seat motion as it happens in a real boat. Adequate performance indicators are superimposed on this scenario in order to enhance the visual feedback to the user. In the upper-left corner of the screen the boat speed, the stroke phase timing, the pace, the last stroke energy input, the distance covered, the last stroke technical efficiency, and the total elapsed time are displayed. Fig. 3 shows a screenshot of typical visual feedback.

\section{DYNAMIC MODELS}

Out-door rowing physics can be modeled focusing on the human dynamics and the hull dynamics. The complexity of such models is established by the degrees of freedom (DoFs) taken into account and the level of detail chosen to model body-fluid interaction. Various studies have attempted to model the human body [10], [17], or the hull-water interaction [3, 15].

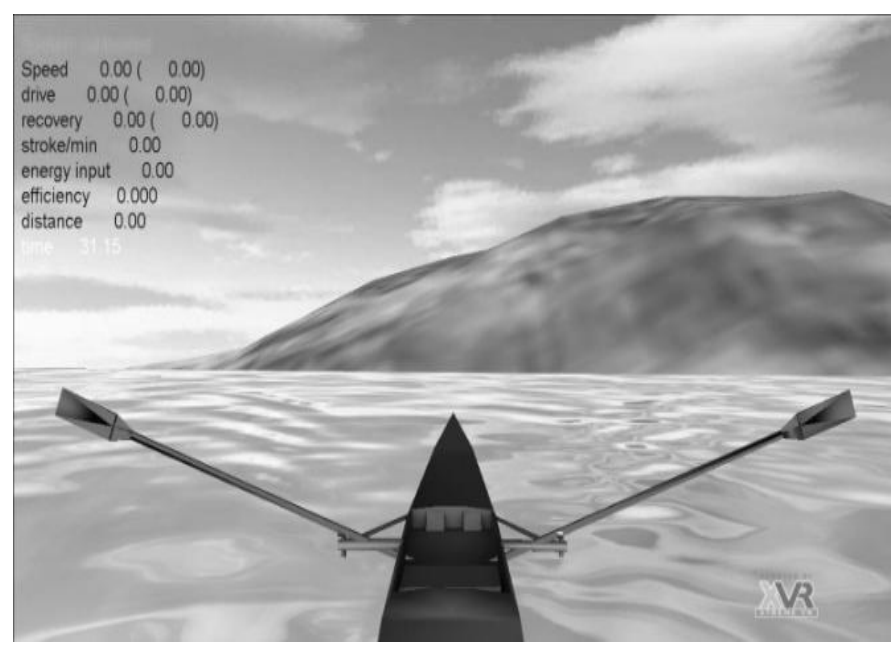

Fig. 3. Screenshot of the VE showing the water inside the simulated landscape and the efficiency parameters plotted in the upper left part of the screen.

The largest part of the rowing dynamic models ([3] for instance), takes into account only the surge of the boat, while the related human models vary from a single mass moving relative to the boat (as in [24]) to two-three DoFs models.
In this paper the first model developed for the SKILLS demonstrator is described and more detailed models are under investigation. The model is composed of three dynamic models: one for the boat, one for the human, and a last one for the oar.

Two main specifications underlie the model development. Models have to detect and penalize the most important technique errors, and they have to be simple enough to allow a real-time simulation.

Since typical technique errors mainly influence the pitch of the boat, we decided to neglect (for the moment) the sway, the roll, and the yaw of the boat. This simplification is not seriously detrimental for intermediate rowers, who are the main target of the demonstrator. As a second hypothesis, we assume the rower movements and exerted forces to be symmetric with respect to the longitudinal symmetry plane of the boat. The same assumption has been made for the Mola, Formaggia, and Miglio [15] and Cabrera, Ruina, and Kleshnev [3] models, as well as other models, and allows the development of 2D models both for the human and hull dynamics. The second hypothesis makes the model more suitable for a sculling crew instead of a sweep rowing one. However, this kind of model allows the simulation of both styles with good results [3]. Another hypothesis has been introduced for a team rowing crew: all teammates are synchronized. This hypothesis makes the model unable to detect team coordination errors, and will be removed in the next model. The advantage of such an hypothesis is that all racing boats can be simulated in the same model, suitably summing the teammates masses and adjusting hull and oar parameters.

\subsection{Hull model}

The hull is considered as a three DoFs rigid body, the free body diagram of the hull is shown in Fig. 4.

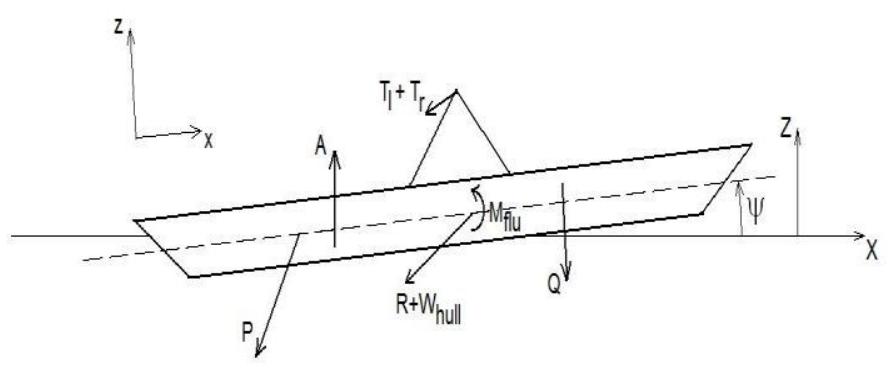

Fig. 4. Hull's free body diagram

The hull DoFs are the surge X, the heave Z, and the pitch $\psi$. The interaction of the rower with the hull is modeled by a force $\mathbf{P}$ at the feet-footstretcher interface and a force $\mathbf{Q}$ on the seat, which is supposed to be square to the seat guide. Since we suppose both the rower and the hull to be symmetric respect to the X-Z plane (Fig. 4), the left and right foot's actions on the foot-stretcher have not been distinguished.

The interaction of the oars with the hull has been modeled by means of the $\mathbf{T}_{\mathrm{r}}$ and $\mathbf{T}_{\mathbf{1}}$ forces. The sum of $\mathbf{T}_{\mathbf{r}}$ and $\mathbf{T}_{\mathbf{1}}$ has been considered in the model, but $\mathbf{T}_{\mathbf{r}}$ and $\mathbf{T}_{\mathbf{l}}$ are separately obtained by the measurement of the force on the two handles and may 
differ a little: this approximation will be removed in the next models.

The hull-fluid interaction has been modeled by means of two forces and a momentum. The A force is due to the Archimende's effect, while the force $\mathbf{R}$ and the momentum $\mathbf{M}_{\text {flu }}$ take into account all the resistant forces due to fluid-dynamics effects such as the wave generation or the friction on the hull's wet surface. While $\mathbf{R}$ is applied in the center of gravity of the hull, $\mathbf{A}$ is applied in the floating center, which is updated step by step during the simulation. $\mathbf{R}$ and $\mathbf{M}_{\mathrm{flu}}$ depend both on the boat

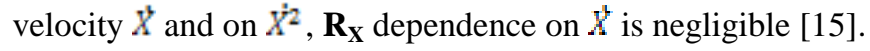

The hull's dynamics are required to determine $\mathbf{P}, \mathbf{Q}$, and $\mathbf{T}$ in order to solve the hull's dynamic equations in $\mathrm{X}, \mathrm{Z}$, and $\psi$. They are determined by means of the human and the oar models.

\subsection{Human model}

The human model is shown in

Fig. 5. It is composed of three rigid bodies representing the calves, the thighs, and all the upper parts of the body (back, head and arms are supposed to compose one rigid body). Feet are supposed to be fixed with respect to the hull, while the pelvis can slide along the hull's longitudinal axis.

Knees and ankles are modeled as rotational joints, therefore the thighs-calves-pelvis compose a one DoF mechanism. The upper part of the body is considered as framed to the pelvis. Hence, such a model does not take into account the back rotation and the arms movements with respect to the body. The whole model has one DoF respect to the hull. Since the seat displacement is measured, the human motion relative to the hull is fully known. Each rigid body mass is supposed to be concentrated in the center of gravity, without momentum of inertia with respect to an axis passing through the center of gravity.

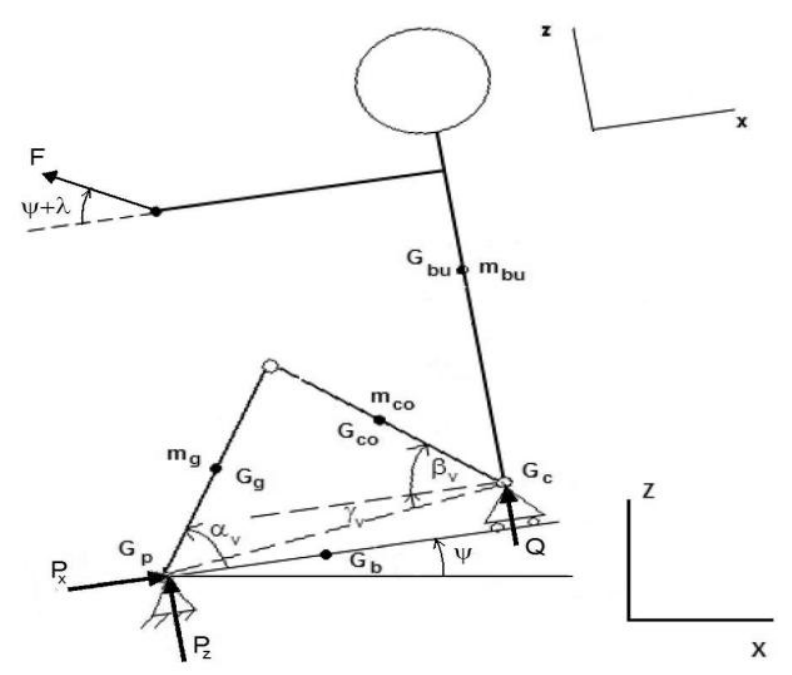

Fig. 5. Human free body diagram

The models under investigation aims at removing the symmetry hypothesis and introducing the back rotation. The forces on the hands, whose sum is $\mathbf{F}$, are measured: therefore, the human dynamics allows the determination of $\mathbf{P}$ and $\mathbf{Q}$ as a function of the hull DoFs.

\subsection{Oar model}

Oars have been modeled as a rigid bar without mass, stressed by the user's force $\mathbf{F}$, the blade water interaction force $\mathbf{C}$ and the contact force at the oar-gate interface $\mathbf{T}$. The free body diagram of the oar is shown in Fig.6.

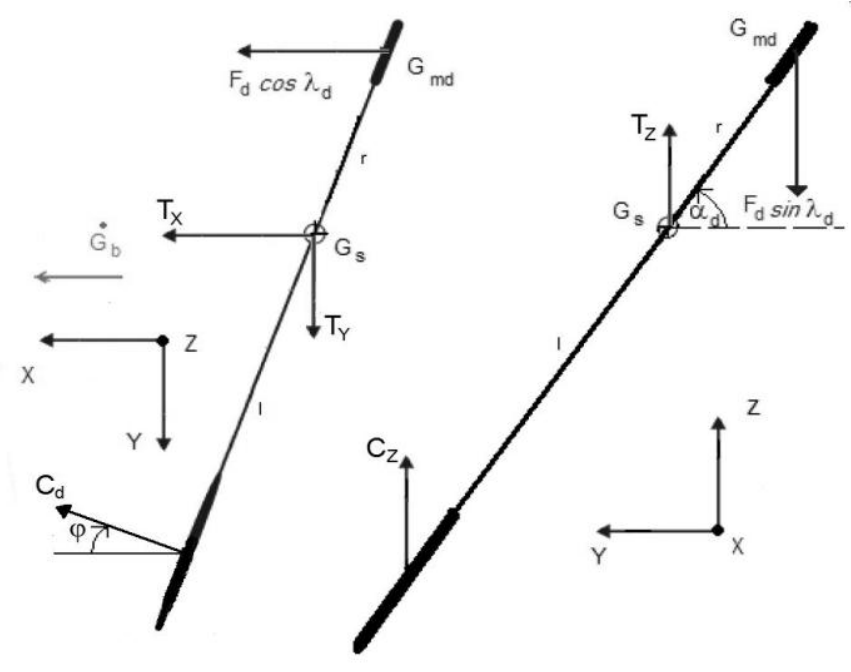

Fig. 6. Oar free body diagram

The component of $\mathbf{C}$ on the X-Y plane $\mathbf{C}_{\mathbf{d}}$ is supposed to be square to the blade, this results from a simplified model of the water-blade interaction. Once $\mathbf{C}_{\mathbf{d}}$ direction is known, $\mathbf{T}$ is determined by the oar balance.

\subsection{Results}

The equations of the three models are grouped in one set of three equations, which is solved in real time. The inputs for such equations are the body motion, the oar kinematics and the forces on the handles. The output is the hull's motion.

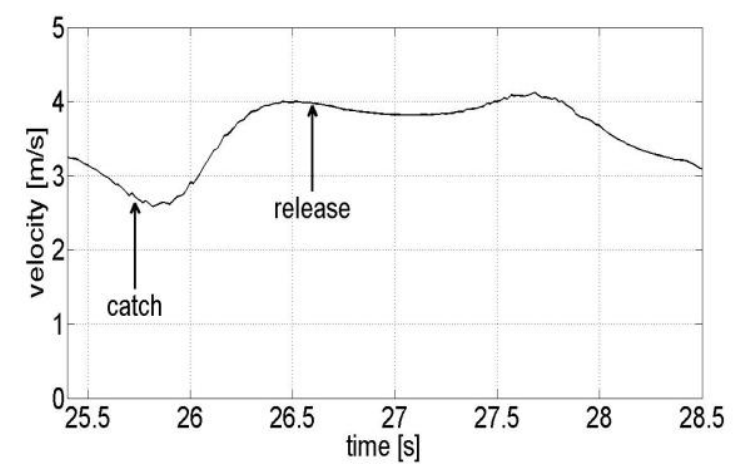

Fig. 7. Hull velocity for a single scull 
This result is very similar to the ones found in literature [3]. The profile (Fig. 7)shows how the inertial effect of the human influence on the system dynamics: the hull reaches maximum velocity during the recovery phase, that is, when the blades are not immersed, but the rower moves toward the boat stern.

\section{MODELING TEAM ROWING}

\subsection{Physics models}

The model described above does not take into account team coordination errors: all mates are supposed to replicate the user's gesture. In point of fact, the mates' misalignment influences the boat dynamics, but its effect is not obvious. For instance, in [12], it is advised to set different positions for the bow and the stern rower in order to reduce the yaw moment due to the different arms of the forces applied on the gates. Even in the sculling boats the effects of a force profiles misalignment on the hull secondary motions (all the motions but the surge) should be analyzed.

However, we suppose the boat to be set-up in order to make a perfect synchronization for the best performance for the crew. Even rowing manuals [13] consider a perfect synchronization to be the goal of the team rowing training, therefore, the training protocol will be developed under such an assumption.

The next model will take into account different mates' behaviors in order to estimate the effect of missing synchronization on the hull's motion.

\subsection{Behavioral model}

Previous researches have shown that the coordination between rhythmic movements of two or more people can be understood in terms of self-organized entrainment processes of coupled oscillators (e.g. [20]). The behaviour of team rowing can be modelled as a coupled-oscillators system where rhythmic movements of each rower are considered as a series of self-sustained oscillators and the multimodal information interaction between them as a coupling function (e.g., [19]). One important question to sort out is which part of the rower movements has to be modelled. This question can be answered by a simple task analysis. The goal of the rowing activity is to move the boat as fast as possible. The only medium rowers use to propel the boat is the oar. The whole intra-coordination behaviour of each rower is focused to enhance the distal movement of the oar. Therefore, the oar movements seem to be the most relevant coupled oscillators to be modelled. We are currently developing a simple model that describes the state of the rowers' synchronization using the oar's movements. In this model, the level of synchronization is characterized by the relative phase of each oar's movement. Using the relative phase of the oar's movement has three main interests. First, the team rowing coordination can be easily characterized by a unique low-dimensional variable. Second, the synchronization is very precisely quantified by the spatio-temporal nature of the relative phase variable (e.g. [22]). Third, the same level of precision is available, regardless of the number rowers and thus, of oars.

\section{FEEDBACK FOR TRAINING}

Many feedback channels are under investigation, in order to find out the best accelerators for learning rowing skills. Visual, acoustic, as well as haptic feedback can be useful for rowing training, and it is worth analyzing their effectiveness as well as the effectiveness of their combination. The most analyzed channel is the visual feedback both for single and for team rowing.

Fig. 3 shows an example of visual feedback for single rowing: such a scenario will be enhanced for team rowing training.

\subsection{Visual feedback about synchronization}

In order to enhance the synchronization, team rowers need to obtain a direct feedback about the level of synchronization among them (e.g., Fig. 8). Fig. 8 is a Lissajous that directly provides information about the team coordination. The abscissa represents the position of the rower's oar and the ordinate corresponds to the virtual (or real) team member's position. The moving curve displays the movements of the rower's oar in function of the team member's position. In fact, it simulates the in-phase coordination average for four cycles [6]. During the learning process the rower will be instructed to pick-up the relevant information from his/her virtual (real) team member visual, acoustic and/or haptic information - so that the moving curve matches as much as possible the bold straight line, representing a pure synchronization between the rower and the team member. The more the rower learns how to synchronize with a team member, the more these two lines match with each other. An ideal synchronization would depict only one line. This kind of biofeedback has already been validated in motor coordination learning in order to learn how to synchronize two joints (for instance the coordination between the hip and the ankle; see [6]).

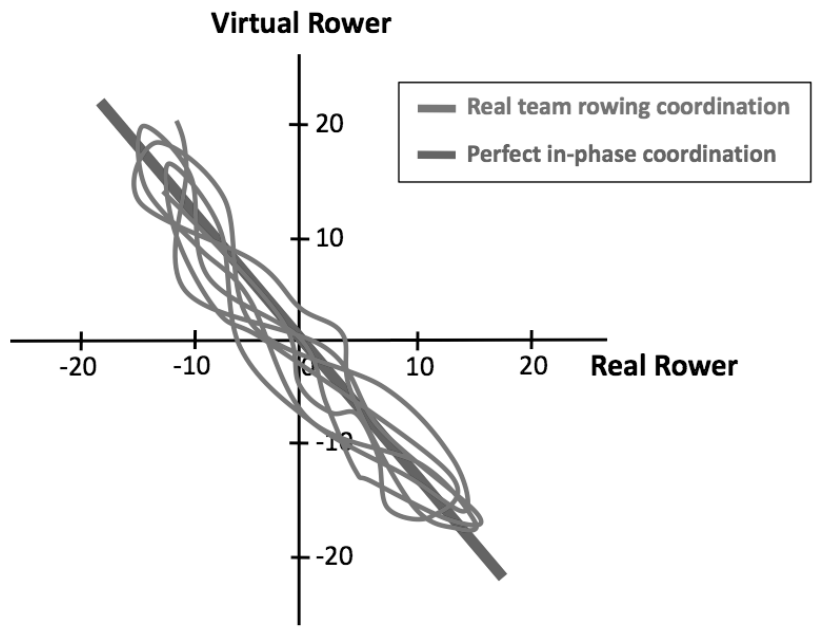

Fig. 8. Example of visual feedback about the coordination between the rower and a virtual partner. The moving curve is the visual feedback indicating the constant coordination between rowers and the bold straight line represents the perfect team rowing synchronization (in phase coordination between rowers). Abscissa and ordinate are in degrees. 
Another type of feedback (closely related with the Lissajous biofeedback), is the relative phase of the oar's movements mentioned above. In the current development of the platform, the simulator displays the continuous curve of the relative phase of each oar's movements on the screen. The relative phase is thus available in real time to each rower. In one experimental validation of this type of feedback, the goal of the rowers is to match their relative phase curves. Another validation being considered is the display of the difference between the two (or more) relative phases of the oar motion, providing a global outcome of the crew synchronization. The purpose of each crew member is to keep the global error curve as minimal as possible, while preserving the general mechanical output. Using such a "collective" visual feedback, we expect rowers to learn faster than when using traditional training protocols of how to pick up the relevant information concerning rower synchronization.

\section{CONCLUSIONS}

Submission of The dynamic models for this rowing training system has been presented. The strategies to implement training protocols for team rowing have been shown, in particular the role of the visual feedback has been underlined for the training of the team.

The next steps of the system development will be an enhancement of the dynamics model and the definition of training protocols. Additional information on this paper is available at http://www.percro.org/papers/vric09row.

\section{REFERENCES}

[1] W. C. Atkinson. Rowing computer research, Available: http://www.atkinsopht.com/row/rowrpage.htm

[2] Biorower. http://www.biorower.com

[3] D. Cabrera, A. Ruina and V. Kleshnev. A simple 1+ dimensional model of rowing mimics observed forces and motion, Human Movement Science, vol. 25, pp. 192-220, 2006.

[4] Concept2. http://www.concept2.com

[5] ETH, $\mathrm{M}^{3}$ rowing simulator, Available: http://www.sms.mavt.ethz.ch/ research/projects/m3/rowing

[6] E. Fugloire, B. G. Bardy and T.A. Stoffregen. (De)stabilization of required abd spontaneous postural dynamics with learning, Journal of Experimental Psychology: Human Perception and Performance, vol. 35, no1, pp.170-187, 2009.

[7] Fédération international des société d'aviron, http://worldrowing.com

[8] A. Frisoli, E. Ruffaldi, L. Bagnoli, A. Filippeschi, C. A. Avizzano, F. Vanni and M. Bergamasco. Preliminary design of rowing simulator for in-door skill training, in 2008 Haptics on ambient system Proc

[9] S.V. Garland. An analysis of pacing strategy adopted by elite competitors in 2000m rowing, Journal of Sports Medicine, , vol. 39, pp. 39-42, 2005.

[10] R. E. Herron, J. R. Cuzzi and J. Hugg. Mass distribution of the human body using biostereometrics, Houston: Texas institute for rehabilitation and research biostereometric laboratory, 1976.

[11] J. Journee. Introduction to ship Hydromechanics, Available: http://www.shipmotions.nl/DUT/LectureNotes/index.html, April 2002.

[12] V. Kleshnev. Rowing biomechanics newslatter, Available: http://www.biorow.com, 2006.

[13] G. La Mura. I fondamenti del canottaggio italiano, Piediluco (Tn) Italy, 1997.

[14] F. Lasome. Rowing Simulator, Available: http://turing.lecolededesign. com/flasorne/portfolio/index.php?2007/06/02/19-rowing-simulator.\%20 $\underline{2007}$

[15] A. Mola, L. Formaggia and E. Miglio. Simulation of the dynamics of an olymoic rowing boat, European Conference on Computational Fluid Dynamics, 2006.
[16] Oartec rower. http://www.oartec.com.au/index.htm

[17] T Robert, L. Chéze, R Dumas and J.P. Verriest. Validation of net joint loads calculated by inverse dynamics in case of complex movements: application to balance recovery movements, Journal of Biomechanics, vol. 40, pp. 2450-2456, 2007.

[18] E. Ruffaldi, A. Filippeschi, A. Frisoli, O. Sandoval, C. A. Avizzano and M. Bergamasco. Vibrotactile perception assessment for a rowing training system, in 2009World HapticsProc.

[19] R.C. Schmidt, C. Carello and M. T. Turvey. Phase transitions and critical fluctuations in visual coordination of rhythmic movements between people, Journal of Experimental Psychology: Human Perception and Performance, vol. 16, pp. 227-247.

[20] R.C. Schmidt and M. J. Richardson. Edited by A. Fuchs and V. Jirsa. Dynamics of interpersonal coordination. Coordination: Neural, Behavioural and Social Dynamics, 2008.

[21] SKILLS. http://www.skills-ip.eu

[22] M. T. Turvey. Coordination, American Psychologist, vol. 45, pp.945-953, 1990.

[23] UCLA marina acquatic center. Rowing Manual, Available: http://marinaaquaticcenter.org/Rowing/Rowing_Manual.pdf

[24] M. Van Holst. On rowing, Available: http://home.hccnet.nl/m.holst/ RoeiWeb.html

[25] J. G. P. Williams. Some biomechanicals aspects of rowing, Rowing: a scientif approach. Kaye and Ward LTD. London, 1967.

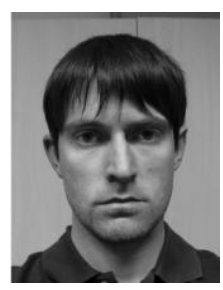

Alessandro Filippeschi (Eng.), is $\mathrm{PhD}$ student in Perceptual Robotics at the PERCRO lab, Scuola Superiore S. Anna, Pisa, Italy. He received a MSc in Mechanical Engineering in 2007, discussing a thesis on the mechanical design of a rowing simulator.

Dr. Filippeschi's current research focuses on analysis, design and implementation of systems for the human motion skills enhancement and/or rehabilitation, it is part of the research activities of the european project SKILLS IP. He is author of 4 papers published in peer-review journals or proceedings.

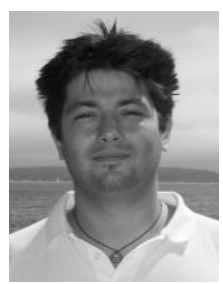

Emanuele Ruffaldi (Eng., PhD) is currently an Assistant Professor in Applied Mechanics of the PERCRO, Scuola Superiore S. Anna, Pisa, Italy. He has obtained his $\mathrm{PhD}$ on Perceptual Robotics in 2006 from Scuola Superiore S.Anna discussing a thesis on perceptually inspired Haptic Algorithms. He received a MSc degree in Computer Engineering in 2002 on a thesis for the visualization of databases based on the metaphor of Information Landscapes. His research interests are in the fields of design of Virtual Reality system in which human, robots and information are integrated. His interaction interests are related to Haptics, in particular haptic rendering, and to the modeling of human skills through intelligent algorithms. He is currently involved in the research activities of the european projects SKILLS IP, ENACTIVE NoE and Decision In Motion STREP. Emanuele has been the author of more than 20 papers published on International Journals and proceedings of scientific workshops. He has been the general chair of the ENACTIVE08 International Conference.

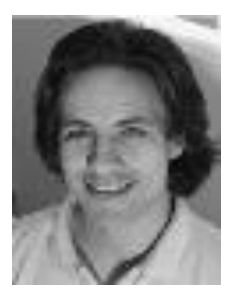

Antonio Frisoli is Assistant Professor of Applied Mechanics at SSSA, Faculty of Engineering, and Head of the Virtual Reality and Telerobotic Systems (VRTS) Research Division of the SSSA laboratory. He received the $\mathrm{PhD}$ in 2002 in Robotics 'summa cum laude' in the area of 'Teleoperation in Virtual Environments' SSSA, the Diploma of Sant' Anna School of University Studies and Doctoral Research (SSSUP), Faculty of Experimental Sciences, Engineering, with full marks in 1998 and the Laurea degree 'summa cum laude' in Mechanical Engineering, specialization in Industrial Automation and Robotics from the University of Pisa in 1998. He teaches the course of 'Mechanics of Robots' at the Faculty of 
Mechanical Engineering of University of Pisa and he is associate member of the ASME and IEEE. He acts as reviewer of several scientific journals and conferences, among them the International Journal of Robotic Research and IEEE Transactions on Robotics and Automation. He is author of more than 50 publications in the area of robotics and virtual reality.

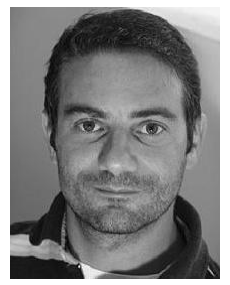

Carlo Alberto Avizzano (Eng., $\mathrm{PhD}$ ) is the Director of the PERCRO Laboratory at Scuola Superiore S.Anna, where he coordinates of research groups on control of haptics and modeling of gestures. Since 2002 Carlo Alberto Avizzano is also external professor at the Faculty of Engineering within the University of Pisa where he teaches the courses of Mechatronics and Industrial Automation Technologies. Carlo Alberto Avizzano research activities deal with intelligent haptics, Teleoperation, Robot control and Autonomous Robots. Main application fields includes: reactive robots, desktop haptics, surgical and rehabilitation robotics, mobile robotics, simulators, technological aids, multimodal augmented tele-learning, design of virtual behaviors and information landscapes. Carlo Alberto Avizzano has coordinated more than 20 National and International projects. He currently participates the activities of the following EU ongoing projects: SKILLS-IP, Presencia, Decision in Motion, NMRS. Carlo Alberto Avizzano has been author of more than 120 papers published on International Journals and proceedings of International peer reviewed scientific conferences. He has been author of 5 different patents and 2 different software copyrights.

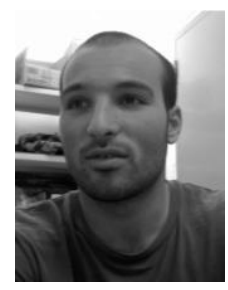

Manuel Varlet, PhD student in Human Movement Science. Ecole doctorale en Science du Mouvement Humain - ${ }^{\circ} 463$ (Human Movement Science) UM1 Motor Efficiency and Deficiency Laboratory manuel.varlet@univ-montp1.fr. He received a MSc in Movement Sciences from the University of the Mediterranean in 2008. His current research focuses on the coordination between people.

Dr. Varlet's most recent publications on international conference are listed below:

- Varlet, M., Marin, L., \& Bardy, B.G. (2008). Postural synchronization in interpersonal visually coupled situation. 10th European Workshop on Ecological Psychology. Madeira, Portugal: November 4-8, 2008.

- Varlet, M., Marin, L., \& Bardy, B.G. (2008). Postural coordination during interpersonal interaction. 5th International Conference on Enactive Interfaces. Pisa, Italy: November 19-21, 2008.

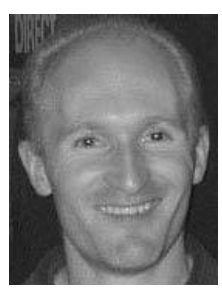

Ludovic Marin, Ph.D., is currently associate Professor (Maître de Conférences Universitaire) in Human Movement Science, of the UM1 - Motor Efficiency and Deficiency Laboratory ludovic.marin@univ-montp1.fr. $\mathrm{He}$ has obtained his PhD in Movement Sciences from University of the Mediterranean in 1998.

$\mathrm{He}$ is Associate Professor of the University of Montpellier-1 since 2001. From 1998 to 2001 he has been Scientific Researcher and supervisor of New York University, NY, USA. From 1997 to 1998 he has been ATER (Temporary Lecturer) of University of Joseph Fourier, Grenoble.

Dr. Marin's main research interests lie in the perception and action field, investigating how controlled movements may be coupled with sensory information. He is particularly interested in skilled behavior in children and adults, both in traditional motor control laboratory tasks and in natural settings. His current research focuses on postural coordination and orientation as well as inter-personal coordination between two (or more) participants. His goal is to determine social sources of inter-personal interactions using limb and postural coordination tasks in different populations. Dr Marin is co-author of more than 40 publications in peer-review journal, books, or proceedings. He is currently involved in several European professional organizations: European Network of Excellence Enactive Interfaces (6th FP), European Integrated Project SKILLS (activity based on interpersonal coordination and on team-rowing demonstrator); Ulysses Program (Ireland-France cooperation) in multimodal social interaction and European Master Program in Sport Psychology.

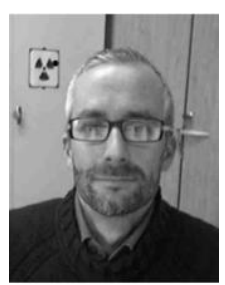

Julien Lagarde, male, 36, is Associate Professor (Maître de Conférences) in Human MovementScience of the University Montpellier1 - Motor Efficiency and Deficiency Laboratory- France julien.lagarde@univ-mtp1.fr/ http://www.edm. univ-montp1.fr/fr/accueil-membre.php?membre $=53 \mathrm{He}$ obtained his PhD in Human Movement Science from University Paul Sabatier in 2001, Toulouse, France. he is Associate Professor of Human Movement Science University Montpellier1since 2007. From 2005 to 2007 he has been Post-doctoral Enactive NE and SKILLS IP from the EC. From 2002 to 2005 he has been Post-doctoral Fellow at the Center for complex systems and brain sciences, Dr. Julien Lagarde investigates multimodal integration in motor control and coordination between individuals. He uses dynamical systems theory and the coordination dynamics framework to understand human adaptive functions, at the behavioral level and at the brain level using non-invasive brain imaging. International recognition Dr Julien Lagarde investigates multimodal integration for motor control and social motor coordination behavior in humans. He uses dynamical systems theory and the coordination dynamics framework to understand human adaptive functions, at the behavioral level and at the brain level using non-invasive brain imaging techniques. He is a member of the European Network of Excellence Enactive Interfaces (6th FP) and the European Integrated Project SKILLS (6th FP). He is the author of more than 12 publications in peer-review journals (e.g., Proc. Natl. Acad. Sci., Experimental Brain Research, Social Neuroscience, etc.) books, or proceedings.

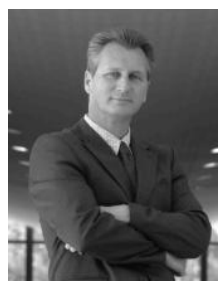

Benoit Bardy (M'76-SM'81-F'87) and the other authors may include biographies at the end of regular papers. Biographies are often not included in conference-related papers. The first paragraph may contain a place and/or date of birth (list place, then date). Next, the author's educational background is listed. The degrees should be listed with type of degree in what field, which institution, city, state or country, and year degree was earned. The author's major field of study should be

lower-cased.

The second paragraph uses the pronoun of the person (he or she) and not the author's last name. It lists military and work experience, including summer and fellowship jobs. Job titles are capitalized. The current job must have a location; previous positions may be listed without one. Information concerning previous publications may be included. Try not to list more than three books or published articles. The format for listing publishers of a book within the biography is: title of book (city, state: publisher name, year) similar to a reference. Current and previous research interests ends the paragraph.

The third paragraph begins with the author's title and last name (e.g., Dr. Smith, Prof. Jones, Mr. Kajor, Ms. Hunter). List any memberships in professional societies other than the IEEE. Finally, list any awards and work for IEEE committees and publications. If a photograph is provided, the biography will be indented around it. The photograph is placed at the top left of the biography. Personal hobbies will be deleted from the biography.

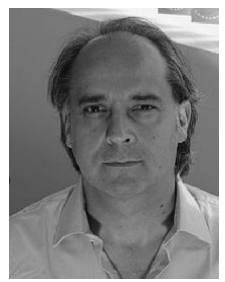

Massimo Bergamasco is Full Professor in Applied Mechanics at the Experimental Science Faculty of Scuola Superiore S. Anna (SSSA), Pisa, Italy. His research activity deals with the study and development of haptic interfaces for the control of the interaction between humans and virtual environments, where he is interested in the kinematics aspects of the design of haptic mechanisms. He has been the Scientific Coordinator of several National projects and of nine EU projects. He has published more than 200 scientific papers in journals and/or international 
conferences proceedings. He is a Member of the Editorial Board of IEEE Computer Graphics and Application, Journal of the VR society, Journal Europèen De Systemes Automatisés and Haptics-e. He is also Member of the Organizing Committee of the following international Conferences: SPIE: Telemanipulator and Telepresence Technologies, IEEE-RO-MAN, EuroHaptics and IEEE WorldHaptics. During his research activities he has already organized the following conferences/workshops: Workshop GLAD-IN-ART, First International Conference on Virtual Environments in Rehabilitation, FIVE 96, RO-MAN 99 and World Haptics Conference 2005. $\mathrm{He}$ is presently the Coordinator of the SKILL IP on capturing and transfer of human skills. 